\title{
ILCEA
}

Revue de l'Institut des langues et cultures

d'Europe, Amérique, Afrique, Asie et Australie

24 | 2015

Lire et écrire ensemble

\section{Une vie de Michel Lafon}

A Life of Michel Lafon

Una vida de Michel Lafon

Julien Roger

\section{OpenEdition}

Journals

Édition électronique

URL : http://journals.openedition.org/ilcea/3554

DOI : 10.4000/ilcea.3554

ISSN : 2101-0609

Éditeur

UGA Éditions/Université Grenoble Alpes

Édition imprimée

ISBN : 978-2-84310-313-1

ISSN : 1639-6073

Référence électronique

Julien Roger, « Une vie de Michel Lafon », ILCEA [En ligne], 24 | 2015, mis en ligne le 02 novembre 2015, consulté le 01 mai 2019. URL : http://journals.openedition.org/ilcea/3554; DOI : 10.4000/ ilcea.3554

Ce document a été généré automatiquement le 1 mai 2019.

(C) ILCEA 


\title{
Une vie de Michel Lafon
}

\author{
A Life of Michel Lafon \\ Una vida de Michel Lafon
}

Julien Roger

Ce sont nos disparus qui nous montrent à vivre (Lafon, 2008:49)

1 Ce qui frappe avant tout lorsque l'on relit l'ensemble de l'œuvre de Michel, c'est surtout sa passion pour la littérature. Comme il le disait quelque temps avant de mourir, sa seule déesse était la littérature (Peeters, 2015: 5). Et, plus particulièrement bien sûr, la littérature argentine. À la fin de la présentation des actes du colloque sur «El escritor argentino y la tradición», en 2004, il qualifiait l'argentinisme de "décentrement au carré »: « [Borges] fonde aussi en grande partie, pour moi, ce décentrement au carré que pourrait être l'argentinisme. Comment ne pas devenir argentiniste, comment ne pas inventer cette nouvelle tradition et ne pas s'y inscrire, quand on reçoit la leçon euphorique que Borges, fantasmatiquement, a ainsi voulu dater de trois décennies à la fois?» (Lafon, $2004:$ 15)

Ou encore, cette phrase dans Une vie de Pierre Ménard: « Reçu ce matin par la poste trois livres d'Argentine, ce pays d'où la lumière ne cesse de me parvenir, cette cocagne littéraire, cet eldorado des fictions jubilatoires et intelligentes. » (Lafon, 2008 : 158)

Michel, c'est peu de l'écrire, était un passionné, qui ne faisait jamais les choses à moitié, en particulier dans la relation qu'il a maintenue avec ses auteurs de prédilection : Borges, Bioy et Aira.

4 Borges, tout d'abord, bien entendu, auquel il consacra ses deux thèses, dont celle d'État, on le sait, publiée dans la collection Poétique du Seuil. Même s'il ne l'a rencontré qu'une fois, il a été, dès le début, ce qui a été à l'origine de sa passion. Borges ne l'a pas quitté pendant toute sa carrière de chercheur, depuis les années 1970 jusqu'à sa mort. Sa maîtrise de traductologie, par exemple, sur César Oudin ${ }^{1}$, était dédiée à Pierre Ménard, comme il l'écrit dans sa position de thèse, après avoir commencé par ces quelques lignes :

Un jour à Montpellier, il y a dix-sept ans, j'ai ouvert pour la première fois un livre

de Borges - c'était El Aleph - et j'ai lu «La casa de Asterión ». J'ai l'impression de 
ne plus être sorti de cette « casa » qui est, comme vous le savez, un labyrinthe et qui aura été pour moi, pour autant que je puisse le dire, un labyrinthe heureux. C'est sans doute ce jour-là que j'ai décidé de lire et d'aimer l'œuvre borgésienne.» (Lafon, 1993 : 109)

Et Michel de conclure sa position de thèse sur un texte de Borges, "La fruición literaria », qu'il choisit tout naturellement de traduire par «Le plaisir du texte »... Passion pour Borges dans son Borges ou la réécriture, bien sûr, qui trouve une dimension génétique et fusionnelle dans l'édition des manuscrits de « Tlön » et « El Sur », en 2010 - la citation est longue, mais elle vaut mieux que toute autre tentative pour décrire la passion de Michel :

Pour l'adolescent qui, il y a presque 40 ans, a découvert les labyrinthes borgésiens et s'y est abîmé avec délice, cette traversée est la réalisation d'un vieux rêve, longtemps caressé. De l'ultime jet de la «première " des fictions aux bifurcations innombrables de la «dernière », du «texte principal» de «Tlön, Uqbar, Orbis Tertius" à son post-scriptum uchronique, de la «discrète narration de faits romanesques » de «El Sur » au dévoilement de son passage secret, c'est une bonne part de la littérature du vingtième siècle qui se joue, une révolution qui s'enclenche, sous la bannière des « œuvres d'imagination raisonnée » et en haine des atermoiements, facilités et autres inconséquences du roman réaliste ou psychologique - comme le proclame ce véritable manifeste poétique qu'est la tonitruante préface de Borges à L'Invention de Morel, en 1940. Mais s'il n'y a pas de livre, dans le monde idéaliste de Tlön, qui ne contienne son propre livre, il n'y a pas non plus de texte borgésien, on le voit, qui ne contienne, d'une façon ou d'une autre, ses avant-textes : ses linéaments, sa préhistoire, sa bibliothèque. Lire Borges (et le traduire, à plus forte raison!) c'est tenter de s'orienter dans cette bibliothèque, c'est inlassablement et savoureusement la «fatiguer ", la hanter. C'est aussi retourner à Adrogué et se perdre une fois de plus dans le grand parc de l'hôtel démoli, entre "vagues statues ", " ruines douteuses ", gloriette, eucalyptus, cour en échiquier et bassins circulaires. J'ai plusieurs fois évoqué, dans les pages qui précèdent, le sentiment paradoxal de longueur extrême qui saisit le lecteur fasciné de ces courtes pièces, indissociable de la jubilation ou de l'angoisse de ne plus jamais en ressortir. Les fictions borgésiennes, si brèves soient-elles en apparence, dialoguent pour l'éternité dans ce parc abandonné, elles s'érigent sur ce roman nostalgique et interminable, que les manuscrits autographes ont sans doute l'immense vertu de déplier comme les images articulées d'un livre d'enfance miraculeusement retrouvé, en restituant à chacune d'entre elles un peu de cette épaisseur romanesque qui reste à jamais son plus beau secret - sa plus belle invention... (Lafon, $2010: 63-64$ )

6 Sa passion pour Bioy, ensuite, se lit dans d'innombrables textes qu'il a écrits sur lui, en particulier, et pour suivre l'ordre chronologique de publication, dans le très beau texte d'introduction à ses Romans, dans la collection Bouquins, chez Robert Laffont :

La fréquentation d'Adolfo Bioy Casares, son regard bleu, son amitié, ses lettres du bout du monde, les retrouvailles à Buenos Aires ou à Paris, les repas au «Lola », la lumière de la Recoleta, les visites rue Posadas, le thé dans le salon de l'immense appartement, au bout des bibliothèques obscures, les confidences des jours fastes et des jours gris, toutes ces heures et tous ces jours restent ineffaçables, pour l'auteur de ces lignes comme pour tous ceux qui ont eu le privilège de l'approcher. (Lafon, $2001:$ L)

7 Passion pour Bioy, enfin, dans la très belle postface à Unos días en el Brasil (Diario de viaje) (Lafon, 2010: 65-82) - nous ne citons pas ici Michel, car c'est toute la postface qu'il faudrait citer, tant chaque ligne respire de cette passion.

8 Aira, enfin, est pour Michel, nous y reviendrons, l'aboutissement de sa carrière, l'ami pour lequel Michel a traduit ses plus beaux textes (nous pensons par exemple à Un épisode 
dans la vie du peintre voyageur ou Varamo, entre autres), qui lui a consacré un colloque (en collaboration avec l'équipe LIRICO), dont le titre des actes est programmatique (César Aira, Une révolution) et qui lui-même a traduit sa Vie de Pierre Ménard.

9 Cette introduction sur la passion qui animait Michel peut sembler convenue, mais elle montre, que Michel était un "fou de littérature ", comme il aimait à le dire à maintes reprises, qu'il s'agisse de littérature argentine, mais aussi de chemins plus rarement fréquentés par la poétique traditionnelle, qu'il s'agisse de la traductologie, de l'écriture en collaboration, ou encore de Tintin, auquel, tout un chacun le sait, Michel a consacré une bonne partie de sa vie. Tintin et Hergé qui apparaissent même dans sa thèse d'État, non pas le volume publié dans la collection Poétique, mais la thèse soutenue à la Sorbonne en 1989 :

Le plus comparable à Borges par la complexité de la pratique qui promeut son œuvre est peut-être Hergé, dont les albums ouvrent leurs fascinants abîmes à la critique récente : Tintin comme seul rival international à Pierre Ménard, le monde borgésien et la planète Borges comme espaces idéaux de la mise en scène et de la mise en œuvre de la question la plus brûlante... (Lafon, 1989 : 525)

Nous n'allons pas, ici, prétendre à expliquer un « système Michel ", un « système Lafon ", car c'est bien l'esprit de système que Michel a combattu toute sa vie, un combat qu'il a mené contre toutes les idées reçues de la littérature traditionnelle, l'œuvre, l'auteur, et ces autres grandes figures que les Nouveaux Critiques ont tant combattu, à commencer par Barthes. Nous nous contenterons de quelques notules sur son œuvre, tant critique que de fiction.

11 Un des grands credo de poéticien de Michel, et qui l'a accompagné toute sa vie (non seulement de chercheur mais aussi de romancier), c'est l'autoréférentialité du texte littéraire. Ou, en d'autres termes, le fait de postuler que l'interprétation, la métatextualité du texte, c'est le texte lui-même. En effet, pour Michel, la transcendance du texte, c'est le texte. D'où cette tautologie : la littérature, c'est la littérature. Le texte n'est pas le miroir de la société qui l'a produit, mais le miroir de lui-même; le texte ne représente, ne symbolise, ne chosifie que lui-même, que sa propre transcendance hypertextuelle. C'est cette idée qui préside à son Borges ou la réécriture, telle que Michel l'énonce en introduction : "Il n'y a, en dernière analyse, ni création, ni innovation, ni découverte : seulement des recréations, des rénovations, des redécouvertes. » (Lafon, 1990 : 23)

Cette idée, très en vogue dans les années 1970, a accompagné Michel toute sa vie, dans la plupart de ses écrits, depuis ses premiers textes. La transcendance textuelle du texte, c'est bien sûr de Ménard que Michel la tient. Sa Vie de Pierre Ménard est une vie de textualité, une vie dans laquelle le texte représente le texte, et non le monde. Même dans le très beau passage sur le plateau du Coiron, en Ardèche, ou dans ce jardin montpelliérain si borgésien, Michel est littéraire, Michel pense à la littérature : le monde est un livre.

Cette entreprise de qualification du texte, autoréférentielle, est ce qui fonde ses premières recherches et qui va trouver ses linéaments jusque dans ses derniers écrits, comme par exemple l'édition génétique de fictions de Borges (Lafon, 2010). Et même plus : elle est, si l'on veut, la pierre angulaire de cette "Vie de Michel Lafon » que nous essayons de dévider. S'il peut exister un quelconque "système Michel Lafon ", c'est bien celui-ci, c'est bien que tout renvoie au Livre, à la littérature, en commençant par la littérature elle-même. 

hommage à une de nos collègues, Carmen Val Julián, se situe, une fois encore, dans la traduction de "Pierre Menard, autor del Quijote " par Paul Verdevoye. Il nous faut citer ici Michel de manière un peu étendue pour mieux comprendre son propos :

Su admirable ambición era producir unas páginas que coincidieran - palabra por palabra y

línea por línea-con las de Miguel de Cervantes.

Son admirable ambition était de reproduire quelques pages qui coïncideraient

- mot pour mot et ligne à ligne - avec celles de Miguel de Cervantès.

Le seul écart apparent est la traduction de producir (produire) par « reproduire ». [... ] Voilà un cas extrême où le simple ajout d'un préfixe verbal peut susciter une contre-lecture de l'ensemble du texte, une lecture extraordinairement appauvrissante, qui, il convient de le signaler, a parfois été faite : celle d'une triste supercherie, d'un titre-canular et d'un Ménard en escroc ou en illusionniste, s'arrangeant pour laisser croire qu'il tire quelques pages du Quichotte de son encrier. [...]

De producir (produire) à « reproduire » [...] il se creuse à l'évidence un décalage, un recul, un retardement. Mon hypothèse est que cet écart notionnel, que le traducteur a sans doute jugé nécessaire d'expliciter, de souligner pour rendre compte de la différence qu'il percevait entre l'activité "productrice » de Cervantès et l'activité «reproductrice» de Ménard, c'est en fait au plus profond, d'une seconde activité reproductrice qu'il parle : celle du traducteur lui-même. Bref, je vois dans cet écart la représentation de l'activité même de celui qui le produit. [...] Cette autoreprésentation, loin d'être exceptionnelle, est la pente quasi naturelle de toute traduction littéraire. » (Lafon, $2011: 335-336)$ puisque c'est de cela qu'il s'agit, sont visibles dans l'hypertexte lui-même. En d'autres termes, l'interprétation du texte ne peut venir que du texte et non pas d'une transcendance extra-textuelle, qui renverrait à un hors-texte. À bien lire ces quelques lignes sur la traduction de "Pierre Ménard", on se prend à regretter amèrement que Michel n'ait pu mener à bien un de ses livres en cours avant sa mort, Traduire Borges.

Un autre domaine de recherches dans lequel Michel a fait preuve de son esprit critique aigu, est l'écriture en collaboration, à laquelle il a consacré ses plus belles pages de chercheur, en collaboration avec Benoît Peeters, avec la publication en 2006, de Nous est un autre (livre auquel il est fait clairement allusion, soit dit en passant, et de manière ludique, dans sa Vie de Pierre Ménard²).

Pour la traduction comme pour l'écriture en collaboration, la poétique du texte est à chercher dans le texte lui-même: nombre de chapitres sur les duos d'écrivains que les auteurs ont mis en scène se terminent par cette conclusion comme celui sur les frères Goncourt, ou à l'image de ces quelques lignes sur Bioy et Borges :

L'écriture en collaboration paraît libérer les inconscients, la dualité et l'oralité convoquant un matériau fantasmatique habituellement refoulé [...]. Cette écriture [...] révèle aussi une tendance massive à parler d'elle-même, à se représenter : au sein de la thématique littéraire proliférante déjà évoquée, le thème de la 
collaboration est omniprésent. [...] Enfin, l'importance du champ lexical de la propriété (paternité, bâtardise, adultère, plagiat, apocryphie, vol, trahison, rivalité...) suggère cette loi : un texte écrit en collaboration parle de son étrange mode de production, l'écriture en collaboration parle de l'écriture en collaboration. (Lafon \& Peeters, $2006: 250$ ) hypertextuelle) est même illustrée par les paratextes étudiés par Michel, notamment dans son ouvrage en collaboration, lorsqu'il étudie, avec Peeters, les différentes couvertures de l'œuvre en collaboration de Borges et de Bioy (Lafon \& Peeters, $2006: 262$ ).

Par ailleurs, à propos de titres, cette propension du titre (et, partant, du texte) à parler de lui-même était déjà en germe dans la thèse d'État de Michel, dans sa partie sur la «titrologie» (c'est ainsi qu'il désignait, presque en même temps que Genette, la paratextualité) ; pour Michel, « tout titre est un métatitre » (Lafon, 1989 : 275) :

Le titre désigne le livre comme livre, le texte comme texte et même l'argument comme argument, l'ébauche comme ébauche. Que l'on pense, pour ces derniers cas, aux nouvelles intitulés « El acercamiento a Almotasim » et « La busca de Averroes », où l'on est ainsi enjoint de lire, par application de ces conjectures, " acercamiento " et «busca » des titres comme incidents non seulement aux acteurs, mais aussi à l'auteur. (Lafon, 1989 : 276)

Que le paratexte réécrive le texte et soit en fait un métatexte, Michel en était convaincu : c'est le sens de ses deux préfaces à L'Invention de Morel, celle de la collection Bouquins et celle de Mourey, l'adaptation du roman de Bioy en bande dessinée. Égalant, voire dépassant Borges (au moins par le nombre), Michel, dans cette dernière préface réécrit littéralement le texte de Bioy qu'il aimait tant. Que le lecteur incrédule (mais le sommesnous vraiment en lisant Michel ?) relise ces quelques lignes de sa préface :

Hoy, en esta isla, ha ocurrido un milagro. Aujourd'hui, dans cette île, un miracle s'est produit [...]. Rarement, me semble-t-il, une œuvre littéraire a fait l'objet d'une adaptation aussi sensible, aussi subtile : c'est la réinvention de Mourey. [...] Si la machine de Morel continue de fonctionner sur une île lointaine du Pacifique, au rythme des marées, espérons qu'elle permet d'enregistrer, pour l'éternité, les passants qui nous sont chers. Ce serait un acte charitable. (Lafon, $2007: 3-4$ )

Une vie de Michel Lafon : une vie de réécrivain, en quelque sorte... comme le suggérait le beau chapitre ainsi intitulé dans son Borges ou la réécriture. (Lafon, 1990 : 73-106)

Mais nous n'en avons pas fini avec la métatextualité inhérente au texte lui-même. Pour Michel, le concept de réécriture, induit par l'autoreprésentativité du texte, était opératoire non plus seulement au niveau d'une poétique, ou d'une critique, mais aussi d'une pragmatique, et d'une pratique. La plus belle illustration de l'hypertextualité, pour Michel, c'est la pratique de la traduction, de Chejfec, de Cervantès, mais aussi et surtout, de Aira. Il y aurait à cet égard un bel article à écrire sur Michel traducteur, un article de traductologie qui viendrait analyser la pratique de la traduction chez Michel. N'ayant pas les compétences pour le faire, nous avons choisi de porter notre regard sur une fiction de Aira traduite par Michel, et qui nous semble à cet égard révélatrice de sa pratique: Varamo.

Le personnage de Varamo, suite à une série de péripéties, écrit, de manière fulgurante, un chef d'œuvre de la littérature et de la poésie d'Amérique centrale, Le Chant de l'Enfant Vierge. Là encore, le texte parle de sa genèse, tout le roman consiste en les prémisses de cette écriture. Et ce texte de Aira n'est pas innocent pour Michel, puisqu'il parle de l'un 
des principaux principes théoriques de Michel : l'interprétation du texte, c'est le texte luimême, le métatexte et le texte ne font qu'un :

Tous les traits circonstanciels dont nous nous sommes servi pour donner couleur et ressemblance au récit de la journée du personnage sont déduits (le mot n'est pas assez fort) du poème qu'il écrivit tout à la fin, et qui constitue le seul document qui soit resté. [...] Une confiance telle que, du texte du poème, on peut déduire le cours des faits qui l'ont précédé, avec une précision qui augmente au fil des lectures successives, et qui ne cesse jamais d'augmenter. [...] Sa capacité à contenir tous les traits circonstanciels préalables à son écriture est un élément historique définitoire. Ce n'est pas parce qu'il s'agit d'une œuvre d'avant-garde qu'elle a ce pouvoir, c'est l'inverse : c'est une œuvre d'avant-garde parce qu'elle permet cette déduction. Autrement dit : est d'avant-garde tout art qui permet de reconstruire les circonstances réelles dans lequel il est né. (Aira, 2005 : 67-69) plus stimulants. Pour qui adopterait un langage genettien, on peut dire que la fiction de Aira traduite par Michel en dit autant sur le roman de Aira que sur la diction, ou plutôt les innombrables dictions de Michel. En somme, la pratique de la traduction chez Michel est le pont entre les dictions poéticiennes, et la fiction, sa Vie de Pierre Ménard. Le choix de Aira, chez Michel, peut sans doute surprendre. En effet, tout semble les opposer, sur le plan de la poétique du texte. Un des aspects fondamentaux de la poétique de Aira est la proclamation maintes fois répétée de la non-réécriture. Le principe de non-réécriture est l'un des piliers de la fiction de Aira : ce dernier a coutume d'écrire qu'il ne se réécrit pas, que le roman B qui suit le roman A est en fait sa réécriture sur le plan fictionnel ${ }^{3}$. On ne trouve pas chez Aira le même moteur de réécriture qui était à l'œuvre chez Borges, ou du moins chez Borges vu par Michel. En d'autres termes, si la poétique de Borges (voire de Bioy Casares, comme il l'écrit au sujet de La Invención de Morel et Plan de evasión) selon Michel devait se lire sous l'angle de la réécriture, il n'y a pas de réécriture, du moins proclamée comme telle, chez Aira. Chaque roman de Aira réécrit sur un plan fictionnel et surtout métaphorique les précédents : c'est en quelque sorte ce principe qui prévaut pour la poétique de Michel. pouvait le faire. Les seules réécritures à l'œuvre dans les écrits de Michel, ce sont les autobiographèmes, notamment celui sur la découverte de «La casa de Asterión ». Pour Michel, les auto-réécritures sont surtout massives, et passent principalement par l'autotraduction (par exemple la très belle postface à Unos días en el Brasil) ou par l'adaptation fondatrice de toute son œuvre, la réécriture de sa thèse d'État qui deviendra son Borges ou la réécriture, publié un an tout juste après la soutenance. Les réécritures sont plus visibles dans son Ménard (voir infra).

La réécriture de sa thèse d'État en essai est à cet égard révélatrice de son travail du texte. Si la thèse d'État était structurée en trois parties (thématique, poétique et efficace de la 
réécriture, Lafon, 1989), son essai est quant à lui une succession de chapitres, sans ordonnancement en grandes parties. De plus, Michel a opéré entre la thèse d'État et Borges ou la réécriture une excision, en termes genettiens: il a supprimé dans l'essai la partie sur les réécritures de Borges par Bioy et Cortázar, bien conscient que cette partie sur l'efficace était, par sa nature même, infinie.

Comme nous l'écrivions dans les lignes qui précèdent, tout fait sens chez Michel, depuis le concept de réécriture borgésienne, jusqu'à la poétique de l'écriture en collaboration, la traductologie, la traduction et la fiction. La fiction, sa Vie de Pierre Ménard, est la continuation logique de son activité de chercheur, et elle est, pourrait-on dire, son pendant naturel, aussi bien sur le plan d'une thématique, jusqu'à la poétique et la fiction.

Mais, avant de nous pencher sur le Michel romancier, il faut rappeler qu'avant d'être auteur de fictions (comme dans son Ménard, mais aussi dans le beau texte de fiction qui clôt Nous est un autre), Michel a été personnage de fiction, sous la plume de Aira, dans Fragmentos de un diario en los Alpes (Aira, 2002). Dans cette novelita, un personnage, spécialiste de Borges et prénommé Michel accueille le narrateur dans sa maison de Voreppe. Clair hommage (au moins au niveau du paratexte) aux Fragments d'un discours amoureux, ce texte, à mi-chemin entre le journal et l'autofiction, raconte un épisode dans la vie de Michel, quelques jours où Aira est venu chez lui. En somme, cette fiction, dans laquelle on lit avec émotion un Michel secret, émouvante au plus haut point, est le passage entre l'activité de poéticien (de chercheur sur la réécriture, sur la traductologie, la traduction) et celle de romancier: Michel sera passé par toutes les étapes, depuis le poéticien, le personnage de roman et le romancier. Tout fait sens, la vie et l'œuvre ne font qu'un, ou ne font qu'une : la passion pour la littérature, le «fou de littérature » qu'il aimait à se décrire.

Le Michel auteur de la fiction est le même Michel poéticien, la fiction sans la poétique n'est pas la fiction, comme en témoigne par exemple le post-scriptum de son roman qui est un roman théorique, un roman poéticien. «Tout, dans une fiction, est fictif », affirmet-il, ou du moins affirme l'instance désignée par «M. L. » (Lafon, $2008: 181$ ), sans au juste que le lecteur ne sache si cette instance est Michel Lafon ou Maurice Legrand, par un ultime pied de nez à Genette et à Borges.

Que la fiction soit une réécriture de la diction et des essais, cela ne fait aucun doute: comment lire autrement les pages sur Ménard traducteur (Lafon, 2008:61 et passim) ? Le narrateur de Une Vie de Pierre Ménard affirme ainsi : « Non, évidemment : je ne copie pas, je ne reproduis pas, je traduis » (Lafon, 2008: 62), qui fait écho à l'article de Michel cité par nous plus haut sur la traduction de "Ménard no quería producir otro Quijote » par «Ménard ne voulait pas reproduire un autre Quichotte 》 (Lafon, 2011 : 335-336).

"Tout, dans une fiction, est fictif » (Lafon, 2008 : 181) : à commencer sans doute par les autobiographèmes de Ménard, et le beau passage sur le Coiron, en Ardèche. Mais cette première fiction publiée par Michel (qui annonce la publication de L'île de Bloy, roman qui, selon sa femme, était terminé) est une fiction sensible, non pas une fiction théorisante (qui serait illisible), mais une fiction traversée par une passion, celle pour Ménard, Borges, Bioy, Valéry, Mallarmé et tant d'autres.

Une vie de réécrivain : ce chapitre du Borges ou la réécriture pourrait s'appliquer également à Une vie de Pierre Ménard, comme le souligne Legrand: "Pour moi, mon siège est fait: nous sommes tous des enfants de Ménard, des réécrivains, inaboutis mais fidèles et 
fascinés » (Lafon, $2008:$ 149). La réécriture, concept central pour Michel, est un principe auquel il a été fidèle toute sa vie de chercheur et de romancier.

Pourquoi lit-on, selon Michel (et Genette) ? Pour les références à la littérature elle-même ; et, en ce sens, le Ménard de Michel accomplit ce que la nouvelle de Borges réclamait depuis sa publication. Nous avons relu bien sûr "Pierre Ménard, auteur du Quichotte ", puis «Le prince de Languedoc au pion provisoirement aboli » (le chapitre consacré à la nouvelle de Borges dans sa thèse, Lafon, 1990 : 51-63) et les allusions à Ménard dans l'œuvre de Michel : la caractéristique la plus frappante de Une vie de Pierre Ménard nous semble être, à l'évidence, son humour, depuis le jeu d'anachronismes délibérés ("L'éditeur, 24 décembre 2008 ", alors que le roman est paru en novembre de cette même année), et les allusions transparentes à une infinité de textes (même si, bien sûr, une partie nous échappe sans doute). $\mathrm{Si}$, un jour, un érudit tente une édition critique et annotée de ce livre, une vie n'y suffira pas... Le plus réussi, parmi tant de belles pages, est sans doute la lettre de Ménard à Borges, magnifique et latéralement réécrite par Borges dans son «Pierre Ménard, auteur du Quichotte »), notamment par l'expression « marbre final» (Lafon, 2008: 168). Nous avons cru initialement que les initiales M. L. du postscriptum renvoyaient à Maurice Legrand, mais il est presque impossible qu'il ait rencontré Borges à Paris en 1983. Mais sans doute est-ce là un anachronisme délibéré de plus? Devons-nous croire que l'instance «M.L.» est la même que le nom sur la couverture? Peut-être, mais alors difficile de comprendre pourquoi de telles justifications, de telles explications qui, dans une certaine mesure, ferment le roman. Tout bien pesé, il est plus savoureux d'attribuer le post-scriptum à Legrand (nous aurions presque préféré qu'il ne soit pas signé). La vie de Ménard (ou plutôt : la vie de Michel avec Ménard), si nous avons bien lu, est un texte fondateur non pas pour ce qu'il annonce mais pour ce qu'il délimite; il pourrait bien être la borne à partir de laquelle Michel aurait pu donner libre carrière à toutes ses velléités de réécriture, fonder toutes les vies de réécrivains, notamment par l'annonce de L'Ile de Bloy (Lafon, 2008: 81) et dont le projet est confirmé dans le post-scriptum (Lafon, 2008 : 181).

Réécriture, ou du moins allusion au livre de Michel et Peeters sur la collaboration (Lafon, 2008: 80), réécriture du «jardin aux sentiers qui bifurquent»(Lafon, 2008: 115), réécriture du prologue sur la fable pour Les Cahiers du Sud (Lafon, 2008: 130 et l'allusion aux numéros de Tigre, la revue fondée par Michel), allusion au football (Lafon, $2008: 122$ ), reprise par Borges de la technique de la réécriture dans son Ménard (Lafon, 2008: 161, "Ménard auteur de Borges auteur de Ménard auteur du Quichotte »), réécriture de " $\mathrm{El}$ Congreso », (Lafon, 2008 : 135-143, explicitée p. 175), l'affirmation soulignée que «Borges eut besoin de Ménard pour devenir Borges... » (Lafon, 2008 : 176).

Mais nous croyons qu'ici il vaut mieux faire confiance à la nostalgie et à la poésie qu'à l'érudition...

En somme, sur bien des points très cachés et, osons le mot, très subtils, qui échapperont à la plupart des lecteurs, dont l'auteur de cet article, la manière dont la fiction prend le relais de la critique ou de la théorie et marque une nouvelle étape, dans la vie de MénardMichel en tout cas mais sans doute aussi dans la vie de beaucoup de poéticiens comme Barthes (Samoyault, 2015: 649-678), la manière aussi dont ce roman joue avec la possibilité et l'impossibilité du roman - puisque à peu près tous les illustres qu'il convoque sont des non-romanciers, voire des irromanciers, ou des romanciers qui renversent le roman, l'absence proclamée de Proust, ou sa présence diffuse, dans les souvenirs méditerranéens, sans parler des contradictions permanentes de Ménard à 
l'égard du roman : une des plus belles découvertes de Michel en romancier aura été qu'un personnage a le droit, et même le devoir, de se contredire. La manière dont cette fiction pourrait être un roman policier où la victime - ou bien le disparu - serait le roman, le risque de «fermeture » du roman par le post-scriptum, l'émotion de la dernière lettre de Ménard à Borges (qui est le socle de la fiction de Borges), la «disparition» finale de Montpellier en même temps que de la fiction et de Legrand (un peu comme à la fin de « La busca de Averroes ») : Michel, à n'en pas douter, avait élaboré là, dans sa fiction, et de manière magistrale, ce que bien de ses dictions avaient mis en théorie.

Et le lecteur se prend à nouveau à regretter de ne pouvoir lire cette Ile de Bloy, maintes fois annoncée dans son Ménard...

On l'aura compris finalement, pour Michel comme pour Proust, Barthes et bien d'autres, la littérature et l'écriture sont le sens même de la vie : c'est l'enseignement qu'il nous a transmis.

Nous laisserons pour finir la parole à Michel, dans ses dernières volontés, telles qu'il les a écrites avant sa mort, sur son bureau, et qui nous semblent la plus belle conclusion possible à cet article :

Si je survis, que ce soit dans les étudiants que j'ai formés, et dans les livres que j'ai écrits. Qu'un étudiant, de temps à autre, ou plus encore un doctorant, pense à moi et parle de moi à ses propres étudiants, à ses propres doctorants, comme je me suis moi-même si souvent référé, en paroles ou en pensées, à mon vieux maître Maurice Molho, et me voilà justifié, perpétué. Que telle ou telle page d'un de mes livres, essais, romans, traductions, éveille le plaisir ou l'intelligence, idem. J'aurai passé une belle partie de ma vie à transmettre le plaisir pris à lire tel ou tel de mes auteurs de chevet, des plus anciens aux plus contemporains, à inculquer à d'autres les enseignements que j'en avais tirés, je crois que j'aurai d'abord et avant tout été un passeur, passionné et parfois, je l'espère, passionnant. Un professeur et un chercheur dignes de ce nom, et dont la lignée, heureusement, se perpétue.

\section{BIBLIOGRAPHIE}

AIRA César (2002), Fragmentos de un diario en los Alpes, Rosario : Beatriz Viterbo.

AIRA César (2005), Varamo, (M. Lafon trad.), Paris : Christian Bourgois.

LAFON Michel (1989), Recherches sur l'œuvre de Jorge Luis Borges (thèse de doctorat d'État), Université Paris-Sorbonne, Paris.

LAFON Michel (1990), Borges ou la réécriture, Paris : Seuil.

LAFON Michel (1993), « Recherches sur l'œuvre de Jorge Luis Borges. Écriture et réécriture (position de thèse) », Sociocriticism, 14, 109-117.

LAFON Michel (2001), « Introduction générale », A. Bioy Casares, Romans (VII-LI), Paris : Robert Laffont. 
LAFON Michel (2004), « À propos de El escritor argentino y la tradición », D. Attala, S. Delgado \& R. Le Marc'Hadour (dir.), L'Écrivain argentin et la Tradition (11-15), Rennes : Presses universitaires de Rennes.

LAFON Michel \& PEETERs Benoît (2006), Nous est un autre. Enquête sur les duos d'écrivains, Paris : Flammarion.

LAFON Michel (2007), « Aujourd'hui, dans cette île... (En guise de préface à L'Invention de Morel) », J.-P. Mourey \& A. Bioy Casares, L'Invention de Morel (3-4), Paris : Casterman.

LAFON Michel (2008), Une vie de Pierre Ménard, Paris : Gallimard.

LAFON Michel (2009), « César Aira », J. Bonnels (dir.), Dictionnaire des écrivains de langue espagnole (15-17), Paris : Robert Laffont.

LAfon Michel (2010), « Posfacio », A. Bioy Casares, Unos días en el Brasil (Diario de viaje) (65-82), Madrid : La Compañía de los libros.

LAFON Michel (2010), «Introduction », J. L. Borges, Deux fictions : «Tlön, Uqbar, Orbis Tertius » et « El Sur» (1-64), adresse de María Kodama. Édition, introduction et traduction de Michel Lafon, Paris : PUF, Genève : Fondation Martin Bodmer.

LAFON Michel (2011), « Menard (acaso sin quererlo). Écrire, traduire, ménardiser », J. Roger, M.L. Ortega \& M. Mestre-Zaragoza (dir.), La realidad y el deseo. Toponymie du découvreur en Amérique espagnole, de Carmen Val Julián, suivi de textes en hommage à l'auteur (331-340), Lyon : ENS Éditions.

PeEters Benoît (2015), « Michel en son absence », B. Peeters (dir.), Hommages à Michel Lafon, (5-6), Bruxelles : Les impressions nouvelles.

SAMOYAULT Thiphaine (2015), Roland Barthes, Paris : Seuil.

\section{NOTES}

1. Oudin qui réapparaît subrepticement dans Une vie de Pierre Ménard: "Imaginez-vous ce que cela signifie, d'être le premier homme à traduire le premier roman de l'ère moderne ? Être le premier, le premier absolument, celui que tous les traducteurs à venir démarqueront d'une manière ou d'une autre [...] ?» (Lafon, 2008: 64). Nous avons choisi dans cet article de citer massivement Michel, car c'est pour nous la meilleure façon de lui rendre hommage, de rendre une partie de son œuvre visible.

2. «La collaboration comme tabou traversant toute la littérature, dont l'étude honnête et minutieuse reste à mener. La fusion de deux je en un nous qui est forcément un autre, à explorer. (Note de l'éditeur) » (Lafon, $2008: 80$ )

3. «Aira prétend ne jamais se relire ni se corriger, préférer le 'nouveau' au 'beau' et se méfier par-dessus tout de la perfection littéraire. Réécrire un roman, pour lui, c'est passer au suivant, dans une 'fuite en avant' qui est, avec le 'continu', la 'miniature' ou le 'sourire sérieux', une des composantes de son 'mythe d'écrivain'. (Lafon, 2009 : 17) 


\section{RÉSUMÉS}

Cet article se veut à la fois un article d'hommage et de mise en perspective de l'œuvre de Michel Lafon, aussi bien en ce qui concerne la poétique que la fiction. Ainsi, on y aborde ses passions pour Borges, Bioy et Aira, ainsi que son activité de traducteur ou de traductologue, et celle de romancier.

This article is both a tribute and a putting into perspective of Michel Lafon's work, both in terms of poetics and fiction. Thus, we approach his passion for Borges, Bioy and Aira and his translator activity or translation studies, and that of the novelist.

Este artículo es a la vez un artículo de homenaje y de puesta en perspectiva de la obra de Michel Lafon, tanto en su poética como en su ficción. Así, estudiamos sus pasiones por Borges, Bioy y Aira y su actividad de traductor y de traductólogo, además de su actividad de novelista.

\section{INDEX}

Palabras claves : Michel Lafon, reescritura, poética, novela

Keywords : Michel Lafon, rewriting, poetics, novel

Mots-clés : Michel Lafon, réécriture, poétique, roman

\section{AUTEUR}

JULIEN ROGER

Université Paris-Sorbonne - Paris 4 